\title{
RELATIVE EFFECTIVENESS OF NEOADJUVANT CHEMOTHERAPY VERSUS PRIMARY SURGERY IN PATIENTS WITH ADVANCED OVARIAN CANCER
}

\author{
Heru Priyanto'), A. Andrijono²) \\ 1)Oncology Division, Department of Obstetrics and Gynecology, Faculty of \\ Medicine, Universitas Sebelas Maret/ Dr. Moewardi Hospital \\ ${ }^{2)}$ Oncology Division, Department of Obstetrics and Gynecology, Faculty of \\ Medicine, Universitas Indonesia/ Dr. Cipto Mangunkusumo Hospital
}

\begin{abstract}
Background: This study aimed to compare the morbidity and disease free progression between patients with advanced ovarian cancer treated with neoadjuvant chemotherapy (NAC) followed by surgery and those treated conventionally with cytoreductive surgery followed by cytotoxic chemotherapy.

Subjects and method: This was a retrospective-cohort study conducted at Dr. Cipto Mangunkusumo Hospital, Jakarta. A sample of 84 patients with advancedstage ovarian cancer was selected for this study, consisting of 64 patients treated conventionally (CT group) with primary surgery followed by platinum-based adjuvant chemotherapy, 20 patients treated with neoadjuvant chemotherapy, of which 8 patients subsequently underwent interval debulking and adjuvant chemotherapy (NAC group). The dependent variables were morbidity and disease free progression. The independent data were neoadjuvant chemotherapy and cytoreductive surgery followed by cytotoxic chemotherapy. The data were collected from medical record and questionnaire. Difference in means between the two groups was tested by t-test. Difference in percents between the two groups was tested by chi-square.

Results: The response rate to the NAC assessed at three cycles was $40 \%$. Performance status ( $\mathrm{Hb}$, albumin, ascites, pleural effusion, $\mathrm{Ca} 125$, and stage) in the NAC group was worse than CT group. Disease free progression after 12 and 24 months in the NAC group was $30 \%$ and $5 \%$, CT group was $10 \%$ and $7.5 \%$, respectively. Parameters of surgical aggressiveness (massive bleeding, organ injury, and ICU stay) were lower in the NAC group than the conventional group. Complication rate of surgical injury in CT group was $17 \%$ and zero in the NAC group.

Conclusion: Neoadjuvant chemotherapy followed by interval debulking in advanced ovarian cancer does not affect disease free progression but reduces morbidity.

Keywords: neoadjuvant chemotherapy, interval debulking, cytoreductive surgery, cytotoxic chemotherapy, disease free progression, morbidity, advanced ovarian cancer.
\end{abstract}

\section{Correspondence:}

Heru Priyanto. Oncology Division, Department of Obstetrics and Gynecology, Faculty of Medicine, Universitas Sebelas Maret/ Dr. Moewardi Hospital, Surakarta, Central Java. Email: drherupriyanto@yahoo.com.

Mobile: 081331529944. 